\title{
Higher Order Mean Squared Error of Generalized Method of Moments Estimators for Nonlinear Models
}

\author{
Yi Hu, ${ }^{1}$ Xiaohua Xia, ${ }^{2}$ Ying Deng, ${ }^{3}$ and Dongmei Guo ${ }^{4}$ \\ ${ }^{1}$ School of Management, University of Chinese Academy of Sciences, Beijing 100190, China \\ ${ }^{2}$ Institute of China's Economic Reform and Development, Renmin University of China, Beijing 100892, China \\ ${ }^{3}$ School of International Trade and Economics, University of International Business and Economics, Beijing 100029, China \\ ${ }^{4}$ School of Economics, Central University of Finance and Economics, Beijing 100081, China \\ Correspondence should be addressed to Dongmei Guo; guodongmeicufe@163.com
}

Received 14 February 2014; Accepted 12 April 2014; Published 28 April 2014

Academic Editor: Chuangxia Huang

Copyright (c) $2014 \mathrm{Yi} \mathrm{Hu}$ et al. This is an open access article distributed under the Creative Commons Attribution License, which permits unrestricted use, distribution, and reproduction in any medium, provided the original work is properly cited.

Generalized method of moments (GMM) has been widely applied for estimation of nonlinear models in economics and finance. Although generalized method of moments has good asymptotic properties under fairly moderate regularity conditions, its finite sample performance is not very well. In order to improve the finite sample performance of generalized method of moments estimators, this paper studies higher-order mean squared error of two-step efficient generalized method of moments estimators for nonlinear models. Specially, we consider a general nonlinear regression model with endogeneity and derive the higher-order asymptotic mean square error for two-step efficient generalized method of moments estimator for this model using iterative techniques and higher-order asymptotic theories. Our theoretical results allow the number of moments to grow with sample size, and are suitable for general moment restriction models, which contains conditional moment restriction models as special cases. The higher-order mean square error can be used to compare different estimators and to construct the selection criteria for improving estimator's finite sample performance.

\section{Introduction}

It is a stylized fact that plenty of relationships are dynamic and nonlinear in nature and society, especially in economic and financial systems [1-8]. These relationships are usually depicted by nonlinear models. Generalized method of moments (GMM) has been widely applied for analysis for these nonlinear models since it was first introduced by Hansen [9] and gradually became a fundamental estimation method in econometrics [10]. Nevertheless, although GMM has good asymptotic properties under fairly moderate regularity conditions, its finite sample performance is not very well [11-13]. Similar to the maximum likelihood estimation (MLE), GMM does not have an exact finite sample distribution. In practice, we generally use the asymptotic distribution to approximate this finite sample distribution, but many applications of GMM reveal that this approximation has low precision [14].
When traditional asymptotic theory cannot precisely approximate the finite sample distributions of estimators or tests, we need higher-order asymptotic expansion for these estimators or tests to get more accurate approximation [15]. Nagar [16] studied the small sample properties of the general $k$-class estimators of simultaneous equations and gave the higher-order asymptotic expansion of the first- and secondorder moments for two-stage least squares (2SLS) estimator. Donald and Newey [17] gave a theoretical derivation of the higher-order MSE for 2SLS based on Nagar [16]; however, their MSE formula applied to the case where the number of instruments grows with but at a smaller rate than the sample size, while Nagar [16] considered the cases where the number of instruments is fixed. Kuersteiner [18] derived the higherorder asymptotic properties of GMM estimators for linear time series models using many lags as instruments.

Besides the linear models, Rilstone et al. [19] derived and examined the second-order bias and MSE of a fairly 
wide class of nonlinear estimators, which included nonlinear least squares, maximum likelihood, and GMM estimators as special cases. Bao and Ullah [20] extended the secondorder bias and MSE results of Rilstone et al. [19] for time series dependent observations. In addition, Bao and Ullah [21] derived the higher-order bias and mean squared error of a large class of nonlinear estimators to order $O\left(n^{-5 / 2}\right)$ and $O\left(n^{-3}\right)$, respectively. However, although these papers gave the high-order bias and MSE for nonlinear estimators, they were not suitable for two-step efficient GMM estimators.

Newey and Smith [22] studied the higher-order bias for two-step GMM estimators, empirical likelihood (EL) and generalized empirical likelihood (GEL) estimators through higher-order asymptotic expansions. But this paper needs to be improved in the following aspects. First, the data generating process considered in this paper was independently identically distributed. Second, the number of moments is fixed. Third, the MSE of GMM was not given. Anatolyev [23] extended Newey and Smith [22] to stationary time series models with serial correlation. Again, the number of moments in this paper was fixed, and this paper only gave the higher-order bias for the estimators, but not the MSE.

Donald et al. [24] examined higher-order asymptotic MSE for conditional moment restriction models. Based on this MSE, they developed moment selection criteria for two-step GMM estimator, a bias corrected version, and GEL estimators. Donald et al. [24] allowed the number of instruments to grow with sample size. However, this paper constructed moment conditions through instrumental variables, which was not suitable for general moment restriction models. Thus, our paper tends to fill an important lacuna in the literature about higher-order asymptotic expansion of nonlinear estimators. Specially, we consider a general nonlinear regression model with endogeneity, and our theoretical results are suitable for general moment restriction models, which contain conditional moment restriction models as a special case.

The remainder of the paper proceeds as follows: Section 2 introduces the model and notations. Section 3 discusses the estimation for the threshold and slope coefficients. Section 4 concludes.

\section{Model}

Many economic and financial models can be written as nonlinear functions of data and parameters. Consider the following nonlinear regression model with endogeneity:

$$
y_{i}=f\left(X_{i}, \beta_{0}\right)+\varepsilon_{i}, \quad i=1, \ldots, n,
$$

where $y_{i}$ is dependent variable, $X_{i}$ is a $p \times 1$ vector of explanatory variables, which contains endogenous variables, $\beta_{0} \in \mathscr{B}$ is a $p \times 1$ vector of parameters, $\mathscr{B}$ is a compact subset of $R^{p}$, and $E\left(\varepsilon_{i}^{2} \mid X_{i}\right)=\sigma_{i}^{2}$.

For model (1), the usual way to estimate $\beta_{0}$ is nonlinear least squares (NLS). However, $X_{i}$ contains endogenous variables, which means that $E\left(\varepsilon_{i} \mid X_{i}\right) \neq 0$. In this case, NLS estimator of $\beta_{0}$ is not consistent. We have to look for another consistent estimator, such as GMM estimator. Let $Z_{i}$ be $L \times 1(L \geq p)$ instrumental variables of $X_{i}$, and $Z_{i}(i=$ $1, \ldots, n)$ are independent random vectors. The orthogonality conditions can be written as

$$
E\left(Z_{i} \varepsilon_{i}\right)=0
$$

And the sample moments are

$$
\begin{aligned}
\bar{g}(\beta) & =\frac{1}{n} \sum_{i=1}^{n} g_{i}(\beta)=\frac{1}{n} \sum_{i=1}^{n} Z_{i} \varepsilon_{i} \\
& =\frac{1}{n} \sum_{i=1}^{n} Z_{i}\left(y_{i}-f\left(X_{i}, \beta\right)\right)=0 .
\end{aligned}
$$

Then, the two-step efficient GMM estimator of $\beta_{0}$ is given by

$$
\widehat{\beta}_{0}=\underset{\beta \in \mathscr{B}}{\arg \min } \bar{g}(\beta)^{\prime} \widetilde{\Phi}(\widetilde{\beta})^{-1} \bar{g}(\beta),
$$

where $\widetilde{\Phi}(\beta)=n^{-1} \sum_{i=1}^{n} g_{i}(\beta) g_{i}(\beta)^{\prime}, \quad \widetilde{\beta}=$ $\arg \min _{\beta \in B} \bar{g}(\beta)^{\prime} \widehat{W}^{-1} \bar{g}(\beta)$, and $\widehat{W}$ is a random weighting matrix that almost surely converges to a nonstochastic symmetric positive definite matrix $W$.

Our goal is to obtain the MSE of $\widehat{\beta}_{0}$. However, formula (4) does not have an analytical solution. We have to obtain it through higher-order asymptotic theory.

\section{Higher-Order MSE of GMM Estimators}

This part uses the iterative idea [19] to derive the higher-order MSE of the GMM estimator, which can be seen as a generalization of Donald et al. [24] to the unconditional moment restriction models. For the convenience of discussion, we use the following notations:

$$
\begin{aligned}
& \Gamma_{j}=\frac{1}{n} \sum_{i=1}^{n} \Gamma_{j i}, \quad \bar{\Gamma}_{j}=\frac{1}{n} \sum_{i=1}^{n} \Gamma_{j i}\left(\beta_{0}\right), \quad \widehat{\Gamma}_{j}=\frac{1}{n} \sum_{i=1}^{n} \Gamma_{j i}\left(\widehat{\beta}_{0}\right), \\
& j=0,1,2, \\
& \underset{\left(L \times p^{j+1}\right)}{\Gamma_{j i}}=E\left(\nabla^{j+1} g_{i}\left(\beta_{0}\right)\right), \quad \Gamma_{j i}(\beta)=\nabla^{j+1} g_{i}(\beta), \\
& \eta_{j i}=\nabla^{j+1} g_{i}\left(\beta_{0}\right)-E\left[\nabla^{j+1} g_{i}\left(\beta_{0}\right)\right]=\Gamma_{j i}\left(\beta_{0}\right)-\Gamma_{j i}, \\
& \Phi=E\left(n^{-1} \sum_{i=1}^{n} g_{i}(\beta) g_{i}(\beta)^{\prime}\right)=\frac{1}{n} \sum_{i=1}^{n} E\left(g_{i}(\beta) g_{i}(\beta)^{\prime}\right),
\end{aligned}
$$

where $\nabla$ is a derivation operator defined by the following recursive method:

$$
\nabla^{r+1} g(\beta)=\left(\frac{\partial\left[\nabla^{r} g(\beta)\right]_{i j}}{\partial \beta^{\prime}}\right)_{\substack{i=1, \ldots, L \\ j=1, \ldots, p^{r}}}
$$

That is, $\nabla^{r+1} g(\beta)$ can be seen as a block matrix whose entry in the $i$ th row and $j$ th column is a $1 \times p$ vector $\partial\left[\nabla^{r} g(\beta)\right]_{i j} / \partial \beta^{\prime}$, 
in which $\left[\nabla^{r} g(\beta)\right]_{i j}$ is the $i$ th row and $j$ th column element of matrix $\nabla^{r} g(\beta)$.

To derive the higher-order expansion of the GMM estimator $\widehat{\beta}_{0}$, the following assumptions for the moment function are required.

Assumption 1. For some neighborhood of $\beta_{0}, f_{i}$ is, at least, three times continuously differentiable and $E\left(\left\|\nabla^{r} f_{i, j}\right\|^{2}\right)<\infty$, $r=0,1,2,3, i=1,2, \ldots$, and $j=1, \ldots, L$.

Assumption 2. For some neighborhood of $\beta_{0}, \| \nabla^{r} g_{i, j}(\beta)-$ $\nabla^{r} g_{i, j}\left(\beta_{0}\right)\|\leq\| \beta-\beta_{0} \| M_{i, j}$, in which $E\left(M_{i, j}\right)<\infty, r=$ $0,1,2,3$, and $j=1, \ldots, L, i=1,2, \ldots$.

Assumption 3. $E\left(\eta_{0 i, j k}^{\tau_{1}} g_{i, r}^{\tau_{2}}\right)=0$, in which $\tau_{1}+\tau_{2}=3, \tau_{1}$ and $\tau_{2}$ are nonnegative integers, $j=1, \ldots, L, k=1, \ldots, p, r=$ $1, \ldots, L$, and $i=1,2, \ldots$.

Assumption 4. The smallest eigenvalues of $E\left[g_{i}\left(\widetilde{\beta}_{0}\right) g_{i}^{\prime}\left(\widetilde{\beta}_{0}\right)\right]$ and $\widetilde{\Phi}\left(\widetilde{\beta}_{0}\right)$ are bounded away from zero, in which $\widetilde{\beta}_{0}$ belongs to the neighborhood of $\beta_{0}$.

Assumption 5. There is $\zeta(L)$ and $\sqrt{L} \leq \zeta(L) \leq C L$ for some finite constant $C$, such that $\left\|g_{i}\right\|<C \zeta(L)$.

Assumption 1 is a necessary condition for a higher-order Taylor expansion. Assumption 2 is a common condition for the moments of remainder terms to bound (see also $[19,20$, 23-25]). Assumption 3 requires that the third moments are zero, which can simplify the MSE calculations (see also [24, $26,27])$. Assumption 4 is a further identification condition. The purpose of Assumption 5 is to control the remainder terms of higher-order expansions (see [25] for details).

The first order condition for the optimization problem in (4) is given by

$$
\left.\left(\frac{\partial \bar{g}(\beta)}{\partial \beta^{\prime}}\right)^{\prime} \widetilde{\Phi}\left(\widetilde{\beta}_{0}\right)^{-1} \bar{g}(\beta)\right|_{\beta=\widehat{\beta}_{0}}=0 .
$$

Define an auxiliary vector $\widehat{\lambda}=-\widetilde{\Phi}\left(\widetilde{\beta}_{0}\right)^{-1} \bar{g}\left(\widehat{\beta}_{0}\right)$; the above first order condition can be rewritten as

$$
\begin{gathered}
\widehat{\Gamma}_{0}^{\prime} \widehat{\lambda}=0, \\
\bar{g}\left(\widehat{\beta}_{0}\right)+\widetilde{\Phi}\left(\widetilde{\beta}_{0}\right) \hat{\lambda}=0 .
\end{gathered}
$$

Let $K=p+L, \widehat{\theta}_{(K \times 1)}=\left(\widehat{\beta}_{0}^{\prime}, \hat{\lambda}^{\prime}\right)^{\prime}$, and $m_{i}(\widehat{\theta})=$ $\left(\begin{array}{c}\hat{\Gamma}_{0 i}^{\prime} \hat{\lambda} \\ g_{i}\left(\widehat{\beta}_{0}\right)+g_{i}\left(\tilde{\beta}_{0}\right) g_{i}\left(\widetilde{\beta}_{0}\right) \hat{\lambda}\end{array}\right)$. Equation (8) can be written as

$$
m(\widehat{\theta})=\frac{1}{n} \sum_{i=1}^{n} m_{i}(\widehat{\theta})
$$

For (9), the number of parameters is equal to the number of equations. A second-order Taylor expansion of (9) at $\theta_{0}=$ $\left(\widehat{\beta}_{0}^{\prime}, 0^{\prime}\right)^{\prime}$ yields

$$
\begin{aligned}
m(\widehat{\theta})= & m+\widehat{M}\left(\widehat{\theta}-\theta_{0}\right)+\frac{1}{2} \sum_{j=1}^{K}\left(\widehat{\theta}_{j}-\theta_{j}\right) \widehat{A}_{j}\left(\widehat{\theta}-\theta_{0}\right) \\
& +\frac{1}{6} \sum_{j=1}^{K} \sum_{k=1}^{K}\left(\widehat{\theta}_{j}-\theta_{j}\right)\left(\widehat{\theta}_{k}-\theta_{k}\right) \widehat{B}_{j k}^{*}\left(\widehat{\theta}-\theta_{0}\right),
\end{aligned}
$$

where $m=m\left(\theta_{0}\right), \widehat{M}=(1 / n) \sum_{i=1}^{n}\left(\partial m_{i}\left(\theta_{0}\right) / \partial \theta^{\prime}\right)=$ $(1 / n) \sum_{i=1}^{n} M_{i}\left(\theta_{0}\right), \widehat{A}_{j}=(1 / n) \sum_{i=1}^{n}\left(\partial M_{i}\left(\theta_{0}\right) / \partial \theta_{j}\right), \widehat{B}_{j k}^{*}=$ $(1 / n) \sum_{i=1}^{n}\left(\partial^{2} M_{i}\left(\theta^{*}\right) / \partial \theta_{j} \partial \theta_{k}\right)$, and $\theta^{*}=t \widehat{\theta}+(1-t) \theta_{0}, t \epsilon$ $(0,1)$, in which $\hat{\theta}_{j}$ and $\theta_{j}$ are the $j$ th element of $\widehat{\theta}$ and $\theta_{0}$, respectively.

From (10), we have

$$
\begin{aligned}
\widehat{\theta}-\theta_{0}= & -M^{-1} m-M^{-1}(\widehat{M}-M)\left(\widehat{\theta}-\theta_{0}\right) \\
& -\frac{1}{2} \sum_{j=1}^{K}\left(\widehat{\theta}_{j}-\theta_{j}\right) M^{-1} A_{j}\left(\widehat{\theta}-\theta_{0}\right)+R, \\
R= & -\frac{1}{2} \sum_{j=1}^{K}\left(\widehat{\theta}_{j}-\theta_{j}\right) M^{-1}\left(\widehat{A}_{j}-A_{j}\right)\left(\widehat{\theta}-\theta_{0}\right) \\
& -\frac{1}{6} \sum_{j=1}^{K} \sum_{k=1}^{K}\left(\widehat{\theta}_{j}-\theta_{j}\right)\left(\widehat{\theta}_{k}-\theta_{k}\right) M^{-1} \widehat{B}_{j k}^{*}\left(\widehat{\theta}-\theta_{0}\right),
\end{aligned}
$$

where $M=E(\widehat{M})$ and $A_{j}=E\left(\widehat{A}_{j}\right)$.

Since the right-hand side of (11) contains terms $\widehat{\beta}_{0}-\beta_{0}$ and $\widehat{\lambda}$, we use iterative techniques to remove these terms. For GMM estimator $\widehat{\beta}_{0}$ under Assumptions $1-5$, if $L \rightarrow \infty$ and $\zeta^{2}(L) L / n \rightarrow 0$, then $\sqrt{n}\left(\widehat{\beta}_{0}-\beta_{0}\right)$ can be decomposed as follows:

$$
\sqrt{n}\left(\widehat{\beta}_{0}-\beta_{0}\right)=\Omega^{-1}\left(h+\sum_{j=1}^{4} T_{j}^{h}+Z^{h}\right),
$$

where

$$
\begin{gathered}
\Omega=\Gamma_{0}^{\prime} \Phi^{-1} \Gamma_{0}, \quad h=-\sqrt{n} \Gamma_{0}^{\prime} \Phi^{-1} \bar{g}=O_{p}(1), \\
T_{1}^{h}=-\sqrt{n}\left(\bar{\Gamma}_{0}-\Gamma_{0}\right)^{\prime} \Sigma \bar{g}=O_{p}\left(\frac{L}{\sqrt{n}}\right), \\
T_{2}^{h}=\sqrt{n} \widehat{\Gamma}_{0}^{\prime} \Phi^{-1} \widetilde{\Phi}_{1} \Sigma \bar{g}=O_{p}\left(\frac{\sqrt{L}}{\sqrt{n}}\right), \\
T_{3}^{h}=T_{31}^{h}+T_{32}^{h}+T_{33}^{h}+T_{34}^{h}+T_{35}^{h}=O_{p}\left(\frac{1}{\sqrt{n}}\right),
\end{gathered}
$$




$$
\begin{aligned}
T_{4}^{h}= & -\sqrt{n} \Gamma_{0}^{\prime} \Phi^{-1} \widetilde{\Phi}_{1} \Sigma \widetilde{\Phi}_{1} \Sigma \bar{g}+\sqrt{n} \Omega^{-1}\left(\bar{\Gamma}_{0}-\Gamma_{0}\right)^{\prime} \Sigma \widetilde{\Phi}_{1} \Sigma \bar{g} \\
= & O_{p}\left(\frac{\zeta(L) L}{n}\right), \\
Z^{h} & =\sqrt{n} \Omega\left(R^{\beta}+R_{1}^{\beta}+R_{2}^{\beta}+R_{3}^{\beta}+R_{4}^{\beta}+R_{5}^{\beta}+R_{6}^{\beta}\right) \\
& =o_{p}\left(\frac{L^{2}}{n}\right),
\end{aligned}
$$

and, for $T_{3}^{h}$,

$$
\begin{gathered}
T_{31}^{h}=\sqrt{n} \Gamma_{0}^{\prime} \Phi^{-1} \widetilde{\Phi}_{\eta g} \Sigma \bar{g} \\
T_{32}^{h}=\sqrt{n} \Gamma_{0}^{\prime} \Phi^{-1}\left(\bar{\Gamma}_{0}-\Gamma_{0}\right) \Omega^{-1} \Gamma_{0}^{\prime} \Phi^{-1} \bar{g} \\
T_{33}^{h}=\frac{1}{2} \sqrt{n} \nabla \Gamma_{0}^{\prime}\left[(\Sigma \bar{g}) \otimes I_{p}\right] \Omega^{-1} \Gamma_{0}^{\prime} \Phi^{-1} \bar{g}, \\
T_{34}^{h}=\frac{1}{2} \sqrt{n}\left[\left(\bar{g}^{\prime} \Phi^{-1} \Gamma_{0} \Omega^{-1}\right) \otimes I_{p}\right] \Gamma_{1}^{\prime} \Sigma \bar{g} \\
T_{35}^{h}=-\frac{1}{2} \sqrt{n} \Gamma_{0}^{\prime} \Phi^{-1} \Gamma_{1}\left[\left(\Omega^{-1} \Gamma_{0}^{\prime} \Phi^{-1} \bar{g}\right) \otimes I_{p}\right] \Omega^{-1} \Gamma_{0}^{\prime} \Phi^{-1} \bar{g} \\
\Sigma=\Phi^{-1}-\Phi^{-1} \Gamma_{0} \Omega^{-1} \Gamma_{0}^{\prime} \Phi^{-1} .
\end{gathered}
$$

Before deriving the higher-order MSE of $\widehat{\beta}_{0}$, we need the following lemmas.

\section{Lemma 6. Consider}

$$
\left(\sum_{j=1}^{4} T_{j}^{h}\right)\left(\sum_{j=1}^{4} T_{j}^{h}\right)^{\prime}=T_{1}^{h} T_{1}^{h^{\prime}}+o_{p}\left(\frac{L^{2}}{n}\right) .
$$

Proof. Consider

$$
\begin{aligned}
\left(\sum_{j=1}^{4} T_{j}^{h}\right)\left(\sum_{j=1}^{4} T_{j}^{h}\right)^{\prime}= & T_{1}^{h} T_{1}^{h^{\prime}}+T_{1}^{h}\left(\sum_{j=2}^{4} T_{j}^{h}\right)^{\prime}+\left(\sum_{j=2}^{4} T_{j}^{h}\right) T_{1}^{h^{\prime}} \\
& +\left(\sum_{j=2}^{4} T_{j}^{h}\right)\left(\sum_{j=2}^{4} T_{j}^{h}\right)^{\prime}
\end{aligned}
$$

in which

$$
\begin{aligned}
T_{1}^{h}\left(\sum_{j=2}^{4} T_{j}^{h}\right)^{\prime}= & O_{p}\left(\frac{L}{\sqrt{n}}\right)\left(O_{p}\left(\frac{\sqrt{L}}{\sqrt{n}}\right)+O_{p}\left(\frac{1}{\sqrt{n}}\right)\right. \\
& \left.+O_{p}\left(\frac{\zeta(L) L}{n}\right)\right) \\
= & O_{p}\left(\frac{L}{\sqrt{n}}\right) O_{p}\left(\frac{\zeta(L) L}{n}\right)
\end{aligned}
$$

$$
\begin{aligned}
& =O_{p}\left(\frac{\zeta(L) L^{2}}{n^{3 / 2}}\right) \\
& =o_{p}\left(\frac{L^{2}}{n}\right) .
\end{aligned}
$$

Similarly, $\left(\sum_{j=2}^{4} T_{j}^{h}\right) T_{1}^{h^{\prime}}=o_{p}\left(L^{2} / n\right)$,

$$
\begin{aligned}
& \left(\sum_{j=2}^{4} T_{j}^{h}\right)\left(\sum_{j=2}^{4} T_{j}^{h}\right)^{\prime} \\
& =\left(O_{p}\left(\frac{\sqrt{L}}{\sqrt{n}}\right)+O_{p}\left(\frac{1}{\sqrt{n}}\right)+O_{p}\left(\frac{\zeta(L) L}{n}\right)\right)^{2} \\
& =O_{p}\left(\frac{\zeta^{2}(L) L^{2}}{n^{2}}\right)=o_{p}\left(\frac{L^{2}}{n}\right) .
\end{aligned}
$$

Lemma 7. Consider

$$
E\left(T_{1}^{h} h^{\prime}\right)=o\left(\frac{L^{2}}{n}\right) .
$$

Proof. By definition, $h=-\sqrt{n} \Gamma_{0}^{\prime} \Phi^{-1} \bar{g}$ and $T_{1}^{h}=$ $-\sqrt{n}\left(\bar{\Gamma}_{0}-\Gamma_{0}\right)^{\prime} \Sigma \bar{g}$; then

$$
\begin{gathered}
T_{1}^{h} h^{\prime}=n\left(\bar{\Gamma}_{0}-\Gamma_{0}\right)^{\prime} \Sigma \bar{g} \cdot \bar{g}^{\prime} \Phi^{-1} \Gamma_{0}, \\
\bar{\Gamma}_{0}-\Gamma_{0}=\frac{1}{n} \sum_{i=1}^{n}\left(\bar{\Gamma}_{0 i}-\Gamma_{0 i}\right)=\frac{1}{n} \sum_{i=1}^{n} \eta_{0 i}, \\
\bar{g} \cdot \bar{g}^{\prime}=\frac{1}{n} \sum_{i=1}^{n} g_{i}\left(\beta_{0}\right) g_{i}^{\prime}\left(\beta_{0}\right)=\frac{1}{n} \sum_{i=1}^{n} g_{i} g_{i}^{\prime},
\end{gathered}
$$

and according to the independence assumption,

$$
\begin{aligned}
E\left(T_{1}^{h} h^{\prime}\right) & =E\left(n\left(\bar{\Gamma}_{0}-\Gamma_{0}\right)^{\prime} \Sigma \bar{g} \cdot \bar{g}^{\prime} \Phi^{-1} \Gamma_{0}\right) \\
& =E\left(n\left(\frac{1}{n} \sum_{i=1}^{n} \eta_{0 i}^{\prime}\right) \Sigma\left(\frac{1}{n} \sum_{i=1}^{n} g_{i} g_{i}^{\prime}\right) \Phi^{-1} \Gamma_{0}\right) \\
& =\frac{1}{n} \sum_{i=1}^{n} E\left(\eta_{0 i}^{\prime} \Sigma g_{i} g_{i}^{\prime}\right) \Phi^{-1} \Gamma_{0} .
\end{aligned}
$$

Let $\eta_{0 i}^{\prime}=A_{(p \times L)}$, and $g_{i} g_{i}^{\prime}=B_{(L \times L)}$; then $\eta_{0 i}^{\prime} \Sigma g_{i} g_{i}^{\prime}=A \Sigma B \triangleq$ $C$. Now, we examine the $j$ th row and $k$ th column element of $C$, $C_{j k}: C_{j k}=\sum_{r=1}^{L} \sum_{s=1}^{L} A_{j r} \Sigma_{r s} B_{s k}=\sum_{r=1}^{L} \sum_{s=1}^{L} \eta_{0 i, r j} \Sigma_{r s} g_{i, s} g_{i, k}$. By Assumption 3, $E\left(C_{j k}\right)=E\left(\sum_{r=1}^{L} \sum_{s=1}^{L} \eta_{0 i, r j} \Sigma_{r s} g_{i, s} g_{i, k}\right)=$ $\sum_{r=1}^{L} \sum_{s=1}^{L} \Sigma_{r s} E\left(\eta_{0 i, r j} g_{i, s} g_{i, k}\right)=0$. Then we have $E(C)=0$; that is, $E\left(\eta_{0 i}^{\prime} \Sigma g_{i} g_{i}^{\prime}\right)=0$. Finally, we have $E\left(T_{1}^{h} h^{\prime}\right)=0$. So, $E\left(T_{1}^{h} h^{\prime}\right)=o\left(L^{2} / n\right)$. 
Lemma 8. Consider

$$
E\left(T_{2}^{h} h^{\prime}\right)=o\left(\frac{L^{2}}{n}\right) .
$$

Proof. By definition of $T_{2}^{h}$ and $h, T_{2}^{h} h^{\prime}=n \Gamma_{0}^{\prime} \Phi^{-1} \widetilde{\Phi}_{1} \Sigma \bar{g}$. $\bar{g}^{\prime} \Phi^{-1} \Gamma_{0}$. And by the definition of $\widetilde{\Phi}_{1}, T_{2}^{h} h^{\prime}=n \Gamma_{0}^{\prime} \Phi^{-1}\left[\widetilde{\Phi}\left(\beta_{0}\right)-\right.$ $\Phi] \Sigma \bar{g} \cdot \bar{g}^{\prime} \Phi^{-1} \Gamma_{0}$. Take mathematical expectation for this formula,

$$
\begin{aligned}
& E\left(T_{2}^{h} h^{\prime}\right) \\
& =E\left(n \Gamma_{0}^{\prime} \Phi^{-1}\left[\widetilde{\Phi}\left(\beta_{0}\right)-\Phi\right] \Sigma \bar{g} \cdot \bar{g}^{\prime} \Phi^{-1} \Gamma_{0}\right) \\
& =n \Gamma_{0}^{\prime} \Phi^{-1} E\left(\left[\widetilde{\Phi}\left(\beta_{0}\right)-\Phi\right] \Sigma \bar{g} \cdot \bar{g}^{\prime}\right) \Phi^{-1} \Gamma_{0} \\
& =n \Gamma_{0}^{\prime} \Phi^{-1} E\left(\frac{1}{n} \sum_{i=1}^{n}\left[g_{i} g_{i}^{\prime}-E\left(g_{i} g_{i}^{\prime}\right)\right] \Sigma\left(\frac{1}{n} \sum_{j=1}^{n} g_{j}\right)\right. \\
& \left.\qquad\left(\frac{1}{n} \sum_{l=1}^{n} g_{l}^{\prime}\right)\right) \Phi^{-1} \Gamma_{0} \\
& =n \Gamma_{0}^{\prime} \Phi^{-1} E\left(\frac{1}{n^{3}} \sum_{i=1}^{n} \sum_{j=1}^{n} \sum_{l=1}^{n}\left[\left[g_{i} g_{i}^{\prime}-E\left(g_{i} g_{i}^{\prime}\right)\right] \Sigma g_{j} g_{l}^{\prime}\right]\right) \Phi^{-1} \Gamma_{0} .
\end{aligned}
$$

According to the independence assumption,

$$
\begin{aligned}
& E\left(\frac{1}{n^{3}} \sum_{i=1}^{n} \sum_{j=1}^{n} \sum_{l=1}^{n}\left[\left[g_{i} g_{i}^{\prime}-E\left(g_{i} g_{i}^{\prime}\right)\right] \Sigma g_{j} g_{l}^{\prime}\right]\right) \\
& =\frac{1}{n^{3}} E\left(\sum_{i=1}^{n}\left\{\left[g_{i} g_{i}^{\prime}-E\left(g_{i} g_{i}^{\prime}\right)\right] \Sigma g_{i} g_{i}^{\prime}\right\}\right) \\
& =\frac{1}{n^{3}} \sum_{i=1}^{n}\left\{E\left(g_{i} g_{i}^{\prime} \Sigma g_{i} g_{i}^{\prime}\right)-E\left(g_{i} g_{i}^{\prime}\right) \Sigma E\left(g_{i} g_{i}^{\prime}\right)\right\} \\
& =\frac{1}{n^{3}} \sum_{i=1}^{n} O(1)=O\left(\frac{1}{n^{2}}\right) .
\end{aligned}
$$

And by $\left\|\Gamma_{0}^{\prime} \Phi^{-1}\right\|=O_{p}(1)$, we have $\Gamma_{0}^{\prime} \Phi^{-1}=O_{p}(1)$. To sum up,

$$
\begin{aligned}
E\left(T_{2}^{h} h^{\prime}\right) & =n O_{p}(1) O_{p}\left(\frac{1}{n^{2}}\right) O_{p}(1) \\
& =O_{p}\left(\frac{1}{n}\right)=o_{p}\left(\frac{L^{2}}{n}\right) .
\end{aligned}
$$

Lemma 9. Consider

$$
E\left(T_{3}^{h} h^{\prime}\right)=o\left(\frac{L^{2}}{n}\right) .
$$

Proof. By definition of $T_{3}^{h}$ and $h, T_{3}^{h} h^{\prime}=\left(T_{31}^{h}+T_{32}^{h}+T_{33}^{h}+\right.$ $\left.T_{34}^{h}+T_{35}^{h}\right) h^{\prime}$.
For the first term, $E\left(T_{31}^{h} h^{\prime}\right)=-n \Gamma_{0}^{\prime} \Phi^{-1} E\left(\widetilde{\Phi}_{\eta g} \Sigma \bar{g} \cdot \bar{g}^{\prime}\right)$ $\Phi^{-1} \Gamma_{0}$.

Since $\widetilde{\Phi}_{\eta g}=\widetilde{\Phi}_{\eta g, 1}+\widetilde{\Phi}_{\eta g, 1}^{\prime}$ and $\widetilde{\Phi}_{\eta g, 1}=$ $(1 / n) \sum_{i=1}^{n} E\left(\eta_{0 i}\left(\widetilde{\beta}_{0}-\beta_{0}\right) g_{i}^{\prime}\right)$, we have $n E\left(\widetilde{\Phi}_{\eta g} \Sigma \bar{g} \cdot \bar{g}^{\prime}\right)=$ $n E\left(\widetilde{\Phi}_{\eta g, 1} \Sigma \bar{g} \cdot \bar{g}^{\prime}\right)+n E\left(\widetilde{\Phi}_{\eta g, 1}^{\prime} \Sigma \bar{g} \cdot \bar{g}^{\prime}\right)$.

According to the independence assumption,

$$
\begin{aligned}
& n E\left(\widetilde{\Phi}_{\eta g, 1} \Sigma \bar{g} \cdot \bar{g}^{\prime}\right) \\
& =n E\left\{\left[\frac{1}{n} \sum_{i=1}^{n} E\left(\eta_{0 i}\left(\widetilde{\beta}_{0}-\beta_{0}\right) g_{i}^{\prime}\right)\right]\right. \\
& \left.\times \sum\left(\frac{1}{n} \sum_{j=1}^{n} g_{j}\right)\left(\frac{1}{n} \sum_{l=1}^{n} g_{l}^{\prime}\right)\right\} \\
& =\frac{1}{n^{2}} \sum_{i=1}^{n} E\left\{\left[E\left(\eta_{0 i}\left(\widetilde{\beta}_{0}-\beta_{0}\right) g_{i}^{\prime}\right)\right] \Sigma g_{i} g_{i}^{\prime}\right\} \\
& +\frac{1}{n^{2}} \sum_{i=1}^{n} \sum_{\substack{j=1 \\
j \neq i}}^{n} E\left\{\left[E\left(\eta_{0 i}\left(\widetilde{\beta}_{0}-\beta_{0}\right) g_{i}^{\prime}\right)\right] \Sigma g_{j} g_{j}^{\prime}\right\} .
\end{aligned}
$$

According to the assumption for $\widetilde{\beta}$ and Assumption 1, the mathematical expectation of the first term is zero.

For the second term, $T_{32}^{h} h^{\prime}=-n \Gamma_{0}^{\prime} \Phi^{-1}\left(\bar{\Gamma}_{0} \quad\right.$ $\left.\Gamma_{0}\right) \Omega^{-1} \Gamma_{0}^{\prime} \Phi^{-1} \bar{g} \cdot \bar{g}^{\prime} \Phi^{-1} \Gamma_{0}$. By Lemma 6, $E\left(T_{32}^{h} h^{\prime}\right)=0$.

For the third term, $T_{33}^{h} h^{\prime}=-(1 / 2) n \nabla \Gamma_{0}^{\prime}[(\Sigma \bar{g}) \otimes$ $\left.I_{p}\right] \Omega^{-1} \Gamma_{0}^{\prime} \Phi^{-1} \bar{g} \cdot \bar{g}^{\prime} \Phi^{-1} \Gamma_{0}$.

For the fourth term, $T_{34}^{h} h^{\prime}=-(1 / 2) n\left[\left(\bar{g}^{\prime} \Phi^{-1} \Gamma_{0} \Omega^{-1}\right) \otimes\right.$ $\left.I_{p}\right] \Gamma_{1}^{\prime} \Sigma \bar{g} \cdot \bar{g}^{\prime} \Phi^{-1} \Gamma_{0}$.

For the fifth term, $T_{35}^{h} h^{\prime}=(1 / 2) n \Gamma_{0}^{\prime} \Phi^{-1} \Gamma_{1}\left[\left(\Omega^{-1} \widehat{\Gamma}_{0}^{\prime} \Phi^{-1} \bar{g}\right) \otimes\right.$ $\left.I_{p}\right] \Omega^{-1} \Gamma_{0}^{\prime} \Phi^{-1} \bar{g} \cdot \bar{g}^{\prime} \Phi^{-1} \Gamma_{0}$.

Similarly, according to the independence assumption and Assumption 1, the mathematical expectation of the third, fourth, and fifth terms are zero.

To sum up, $E\left(T_{3}^{h} h^{\prime}\right)=o\left(L^{2} / n\right)$.

Lemma 10. Consider

$$
E\left(T_{4}^{h} h^{\prime}\right)=o\left(\frac{L^{2}}{n}\right)
$$

Proof. By definition of $T_{4}^{h}$ and $h$,

$$
\begin{aligned}
T_{4}^{h} h^{\prime}= & n \Gamma_{0}^{\prime} \Phi^{-1} \widetilde{\Phi}_{1} \Sigma \widetilde{\Phi}_{1} \Sigma \bar{g} \cdot \bar{g}^{\prime} \Phi^{-1} \Gamma_{0} \\
& -n \Omega^{-1}\left(\bar{\Gamma}_{0}-\Gamma_{0}\right)^{\prime} \Sigma \widetilde{\Phi}_{1} \Sigma \bar{g} \cdot \bar{g}^{\prime} \Phi^{-1} \Gamma_{0} .
\end{aligned}
$$

Similar to the proof of Lemma 8, we have

$$
E\left(n \Gamma_{0}^{\prime} \Phi^{-1} \widetilde{\Phi}_{1} \Sigma \widetilde{\Phi}_{1} \Sigma \bar{g} \cdot \bar{g}^{\prime} \Phi^{-1} \Gamma_{0}\right)=o\left(\frac{L^{2}}{n}\right)
$$


And according to the independence assumption and Assumption 1,

$$
E\left(-n \Omega^{-1}\left(\bar{\Gamma}_{0}-\Gamma_{0}\right)^{\prime} \Sigma \widetilde{\Phi}_{1} \Sigma \bar{g} \cdot \bar{g}^{\prime} \Phi^{-1} \Gamma_{0}\right)=0=o\left(\frac{L^{2}}{n}\right)
$$

To sum up, $E\left(T_{4}^{h} h^{\prime}\right)=o\left(L^{2} / n\right)$.

Using these lemmas, then we can get the higher-order MSE of $\widehat{\beta}_{0}$ as follows.

Theorem 11. For GMM estimator $\widehat{\beta}_{0}$ in (4), under Assumptions 1-5, if $L \rightarrow \infty$ and $\zeta^{2}(L) L / n \rightarrow 0$, then the higher-order MSE of $\sqrt{n} \widehat{\beta}_{0}$ is given by

$$
\operatorname{MSE}\left(\sqrt{n} \widehat{\beta}_{0} \mid Z\right)=\Omega^{-1}+\Omega^{-1} \frac{\Pi^{\prime}}{n} \Omega^{-1}+o\left(\frac{L^{2}}{n}\right)
$$

in which

$$
\begin{gathered}
\Omega^{-1}=n\left[E(F \mid Z)^{\prime} Z\left(\sum_{i=1}^{n} \sigma_{i}^{2} Z_{i} Z_{i}^{\prime}\right)^{-1} Z^{\prime} E(F \mid Z)\right]^{-1} \\
\Pi=\sum_{i=1}^{n} B_{i i} \sigma_{u \varepsilon, i} \\
\Gamma_{0}=\frac{1}{n} \sum_{i=1}^{n} \Gamma_{0 i}=\frac{1}{n} \sum_{i=1}^{n} E\left(-Z_{i} \frac{\partial f_{i}}{\partial \beta^{\prime}} \mid Z_{i}\right) \\
=-\frac{1}{n} Z^{\prime} E(F \mid Z) \\
F=\left(\frac{\partial f_{1}}{\partial \beta}, \ldots, \frac{\partial f_{n}}{\partial \beta}\right)^{\prime} \\
\Phi=E\left(\frac{1}{n} \sum_{i=1}^{n} g_{i} g_{i}^{\prime} \mid Z\right) \\
u_{i}=\frac{\partial f_{i}}{\partial \beta}-E\left(\frac{\partial f_{i}}{\partial \beta} \mid Z_{i}\right) \\
=\frac{1}{n} E\left(\sum_{i=1}^{n} Z_{i} \varepsilon_{i} \varepsilon_{i} Z_{i}^{\prime} \mid Z\right) \\
\sigma_{u \varepsilon, i}^{n} \sigma_{i}^{2} Z_{i} Z_{i}^{\prime}, \\
B_{i i}=\frac{Z_{i}^{\prime} \Sigma Z_{i}}{n}, \\
\Gamma_{0} \Omega^{-1} \Gamma_{0}^{\prime} \Phi^{-1}
\end{gathered}
$$

In addition, by definition of $T_{1}^{h}, T_{1}^{h} T_{1}^{h^{\prime}}=n\left(\bar{\Gamma}_{0}-\Gamma_{0}\right)^{\prime} \Sigma \bar{g}$. $\bar{g}^{\prime} \Sigma\left(\bar{\Gamma}_{0}-\Gamma_{0}\right)$. Consider

$$
\begin{aligned}
\bar{\Gamma}_{0}-\Gamma_{0} & =\frac{1}{n} \sum_{i=1}^{n}\left(\bar{\Gamma}_{0 i}-\Gamma_{0 i}\right) \\
& =-\frac{1}{n} \sum_{i=1}^{n} Z_{i}\left[\frac{\partial f_{i}}{\partial \beta^{\prime}}-E\left(\frac{\partial f_{i}}{\partial \beta^{\prime}} \mid Z_{i}\right)\right] .
\end{aligned}
$$

Denote $u_{i}=\left(\partial f_{i} / \partial \beta\right)-E\left(\left(\partial f_{i} / \partial \beta\right) \mid Z_{i}\right)$; then $\bar{\Gamma}_{0}-\Gamma_{0}=$ $-(1 / n) Z^{\prime} u . \bar{g}=(1 / n) \sum_{i=1}^{n} g_{i}=(1 / n) \sum_{i=1}^{n} Z_{i} \varepsilon_{i}=(1 / n) Z^{\prime} \varepsilon$. Then,

$$
\begin{aligned}
T_{1}^{h} T_{1}^{h^{\prime}} & =n\left(-\frac{1}{n} Z^{\prime} u\right)^{\prime} \Sigma\left(\frac{1}{n} Z^{\prime} \varepsilon \frac{1}{n} \varepsilon^{\prime} Z\right) \Sigma\left(-\frac{1}{n} Z^{\prime} u\right) \\
& =\frac{1}{n} u^{\prime} \frac{Z \Sigma Z^{\prime}}{n} \varepsilon \varepsilon^{\prime} \frac{Z \Sigma Z^{\prime}}{n} u=\frac{1}{n} u^{\prime} B \varepsilon \varepsilon^{\prime} B u .
\end{aligned}
$$


According to the independence assumption,

$$
\begin{aligned}
& E\left(T_{1}^{h} T_{1}^{h^{\prime}} \mid Z\right) \\
& =\frac{1}{n} E\left(\sum_{i, j, k, l} u_{i} B_{i j} \varepsilon_{j} \varepsilon_{k} B_{k l} u_{l}^{\prime} \mid Z\right) \\
& =\frac{1}{n} E \\
& \times\left(\left\{\sum_{i=j=k=l}+\sum_{i=j=k \neq l}+\sum_{i=j \neq k=l}+\sum_{i=j \neq k \neq l}\right.\right. \\
& \left.+\sum_{i \neq j=k=l}+\sum_{i \neq j=k \neq l}+\sum_{i \neq j \neq k=l}+\sum_{i \neq j \neq k \neq l}\right\} \\
& \left.\times u_{i} B_{i j} \varepsilon_{j} \varepsilon_{k} B_{k l} u_{l}^{\prime} \mid Z\right) \\
& =\frac{1}{n} E\left(\sum_{i=1}^{n} u_{i} B_{i i} \varepsilon_{i} \varepsilon_{i} B_{i i} u_{i}^{\prime} \mid Z\right) \\
& +\frac{1}{n} E\left(\sum_{i \neq k} u_{i} B_{i i} \varepsilon_{i} \varepsilon_{k} B_{k k} u_{k}^{\prime} \mid Z\right) \\
& +\frac{1}{n} E\left(\sum_{i \neq j} u_{i} B_{i j} \varepsilon_{j} \varepsilon_{j} B_{j i} u_{i}^{\prime} \mid Z\right) \\
& +\frac{1}{n} E\left(\sum_{i \neq j} u_{i} B_{i j} \varepsilon_{j} \varepsilon_{i} B_{i j} u_{j}^{\prime} \mid Z\right) \\
& =\frac{1}{n} \sum_{i=1}^{n} B_{i i}^{2} E\left(u_{i} \varepsilon_{i} \varepsilon_{i} u_{i}^{\prime} \mid Z\right) \\
& +\frac{1}{n} \sum_{i \neq k} B_{i i} B_{k k} E\left(u_{i} \varepsilon_{i} \mid z_{i}\right) E\left(\varepsilon_{k} u_{k}^{\prime} \mid z_{k}\right) \\
& +\frac{1}{n} \sum_{i \neq j} B_{i j}^{2} E\left(u_{i} u_{i}^{\prime} \mid z_{i}\right) E\left(\varepsilon_{j}^{2} \mid z_{j}\right) \\
& +\frac{1}{n} \sum_{i \neq j} B_{i j}^{2} E\left(u_{i} \varepsilon_{i} \mid z_{i}\right) E\left(\varepsilon_{j} u_{j}^{\prime} \mid z_{j}\right) \\
& =\frac{1}{n}\left[\sum_{i=1}^{n} B_{i i} \sigma_{u \varepsilon, i}\right]\left[\sum_{i=1}^{n} B_{i i} \sigma_{u \varepsilon, i}\right]^{\prime}+o_{p}\left(\frac{L^{2}}{n}\right) \\
& =\frac{\Pi \Pi^{\prime}}{n}+o_{p}\left(\frac{L^{2}}{n}\right) \text {. }
\end{aligned}
$$

In (32), $\Omega^{-1}$ is asymptotic variance of $\sqrt{n} \widehat{\beta}_{0}$, and $\Omega^{-1}\left(\Pi \Pi^{\prime} / n\right) \Omega^{-1}$ can be seen as the asymptotic bias terms. In practice, $\Omega$ and $\Pi$ can be substituted by their consistent estimators.

\section{Conclusions}

In this paper, we consider a general nonlinear regression model with endogeneity and derive the higher-order mean square error of two-step efficient generalized method of moments estimators for this nonlinear model. The theoretical results in this paper allow the number of moments to grow with but at smaller rate than the sample size. And the derivations are suitable for general moment restriction models, which contain conditional moment restriction models and linear models as special cases. The higher-order mean squared error got in this paper has many uses. For example, it can be used to compare among different estimators or to construct the selection criteria of moments for improving the finite sample performance of GMM estimators. This paper considered a restrictive condition in which the data generating process is independent. It would be valuable to extend the results to the dynamic panel data models, in which the moments are going with the time dimension. It is saved for future research.

\section{Conflict of Interests}

The authors declare that there is no conflict of interests regarding the publication of this paper.

\section{Acknowledgments}

This work was partially supported by the National Natural Science Foundation of China (Grant nos. 71301160, 71203224, and 71301173), Beijing Planning Office of Philosophy and Social Science (13JGB018), and Program for Innovation Research in Central University of Finance and Economics.

\section{References}

[1] A. Ciarreta and A. Zarraga, "Economic growth-electricity consumption causality in 12 European countries: a dynamic panel data approach," Energy Policy, vol. 38, no. 7, pp. 3790-3796, 2010.

[2] O. Galor and D. N. Weil, "Population, technology, and growth: from malthusian stagnation to the demographic transition and beyond," The American Economic Review, vol. 90, no. 4, pp. 806$828,2000$.

[3] Y. Hu, X.-H. Xia, M.-J. Wang, and Z.-M. Chen, "The effects of cross-section dimension $n$ in panel co-integration test," Communications in Nonlinear Science and Numerical Simulation, vol. 15, no. 4, pp. 1019-1027, 2010.

[4] C.-X. Huang, C.-L. Peng, X.-H. Chen, and F.-H. Wen, "Dynamics analysis of a class of delayed economic model," Abstract and Applied Analysis, vol. 2013, Article ID 962738, 12 pages, 2013.

[5] C.-X. Huang, X. Gong, X.-H. Chen, and F.-H. Wen, "Measuring and forecasting volatility in Chinese stock market using HARCJ-M model," Abstract and Applied Analysis, vol. 2013, Article ID 143194, 13 pages, 2013.

[6] J. H. Lin, Y. Hu, M. J. Wang, and X. H. Xia, "A Monte Carlo comparison of panel unit root tests under factor structure," Applied Economics Letters, vol. 20, no. 3, pp. 288-291, 2013.

[7] F. Wen and Z. Liu, "A copula-based correlation measure and its application in chinese stock market," International Journal 
of Information Technology \& Decision Making, vol. 8, no. 4, pp. 787-801, 2009.

[8] F. Wen, Z. Li, C. Xie, and D. Shaw, "Study on the fractal and chaotic features of the Shanghai composite index," Fractals, vol. 20, no. 2, pp. 133-140, 2012.

[9] L. P. Hansen, "Large sample properties of generalized method of moments estimators," Econometrica, vol. 50, no. 4, pp. 10291054, 1982.

[10] Y. Hu, D.-M. Guo, Y. Deng, and S.-Y. Wang, "Estimation of nonlinear dynamic panel data models with individual effects," Mathematical Problems in Engineering, vol. 2014, Article ID 672610, 7 pages, 2014.

[11] B. Antoine and E. Renault, "Efficient GMM with nearly-weak instruments," The Econometrics Journal, vol. 12, supplement s1, pp. S135-S171, 2009.

[12] M. Caner, "Lasso-type GMM estimator," Econometric Theory, vol. 25, no. 1, pp. 270-290, 2009.

[13] I. A. Canay, "Simultaneous selection and weighting of moments in GMM using a trapezoidal kernel," Journal of Econometrics, vol. 156, no. 2, pp. 284-303, 2010.

[14] W. K. Newey and F. Windmeijer, "Generalized method of moments with many weak moment conditions," Econometrica, vol. 77, no. 3, pp. 687-719, 2009.

[15] J. F. Kiviet and G. D. A. Phillips, "Higher-order asymptotic expansions of the least-squares estimation bias in first-order dynamic regression models," Computational Statistics \& Data Analysis, vol. 56, no. 11, pp. 3705-3729, 2012.

[16] A. L. Nagar, "The bias and moment matrix of the general $k$ class estimators of the parameters in simultaneous equations," Econometrica, vol. 27, no. 4, pp. 575-595, 1959.

[17] S. G. Donald and W. K. Newey, "Choosing the number of instruments," Econometrica, vol. 69, no. 5, pp. 1161-1191, 2001.

[18] G. M. Kuersteiner, "Kernel-weighted GMM estimators for linear time series models," Journal of Econometrics, vol. 170, no. 2, pp. 399-421, 2012.

[19] P. Rilstone, V. K. Srivastava, and A. Ullah, "The second-order bias and mean squared error of nonlinear estimators," Journal of Econometrics, vol. 75, no. 2, pp. 369-395, 1996.

[20] Y. Bao and A. Ullah, "The second-order bias and mean squared error of estimators in time-series models," Journal of Econometrics, vol. 140, no. 2, pp. 650-669, 2007.

[21] Y. Bao and A. Ullah, "Higher-order bias and MSE of nonlinear estimators," Pakistan Journal of Statistics, vol. 25, no. 4, pp. 587594, 2009.

[22] W. K. Newey and R. J. Smith, "Higher order properties of GMM and generalized empirical likelihood estimators," Econometrica, vol. 72, no. 1, pp. 219-255, 2004.

[23] S. Anatolyev, "GMM, GEL, serial correlation, and asymptotic bias," Econometrica, vol. 73, no. 3, pp. 983-1002, 2005.

[24] S. G. Donald, G. W. Imbens, and W. K. Newey, "Choosing instrumental variables in conditional moment restriction models," Journal of Econometrics, vol. 152, no. 1, pp. 28-36, 2009.

[25] S. G. Donald, G. W. Imbens, and W. K. Newey, "Empirical likelihood estimation and consistent tests with conditional moment restrictions," Journal of Econometrics, vol. 117, no. 1, pp. 55-93, 2003.

[26] J. Alvarez and M. Arellano, "The time series and cross-section asymptotics of dynamic panel data estimators," Econometrica, vol. 71, no. 4, pp. 1121-1159, 2003.

[27] R. Okui, "The optimal choice of moments in dynamic panel data models," Journal of Econometrics, vol. 151, no. 1, pp. 1-16, 2009. 


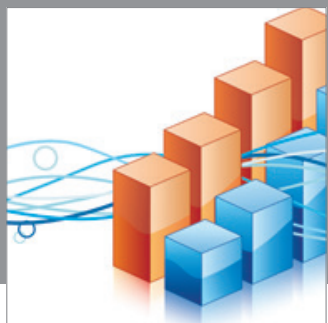

Advances in

Operations Research

mansans

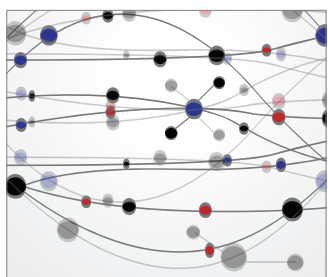

The Scientific World Journal
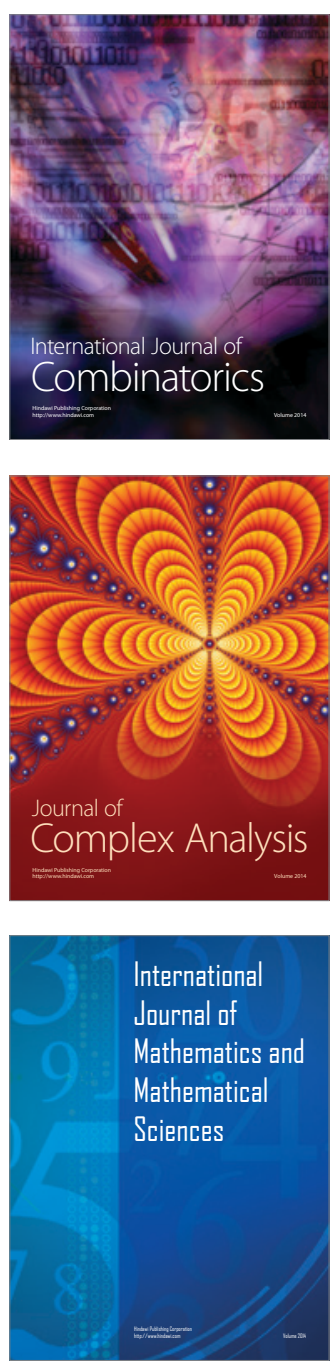
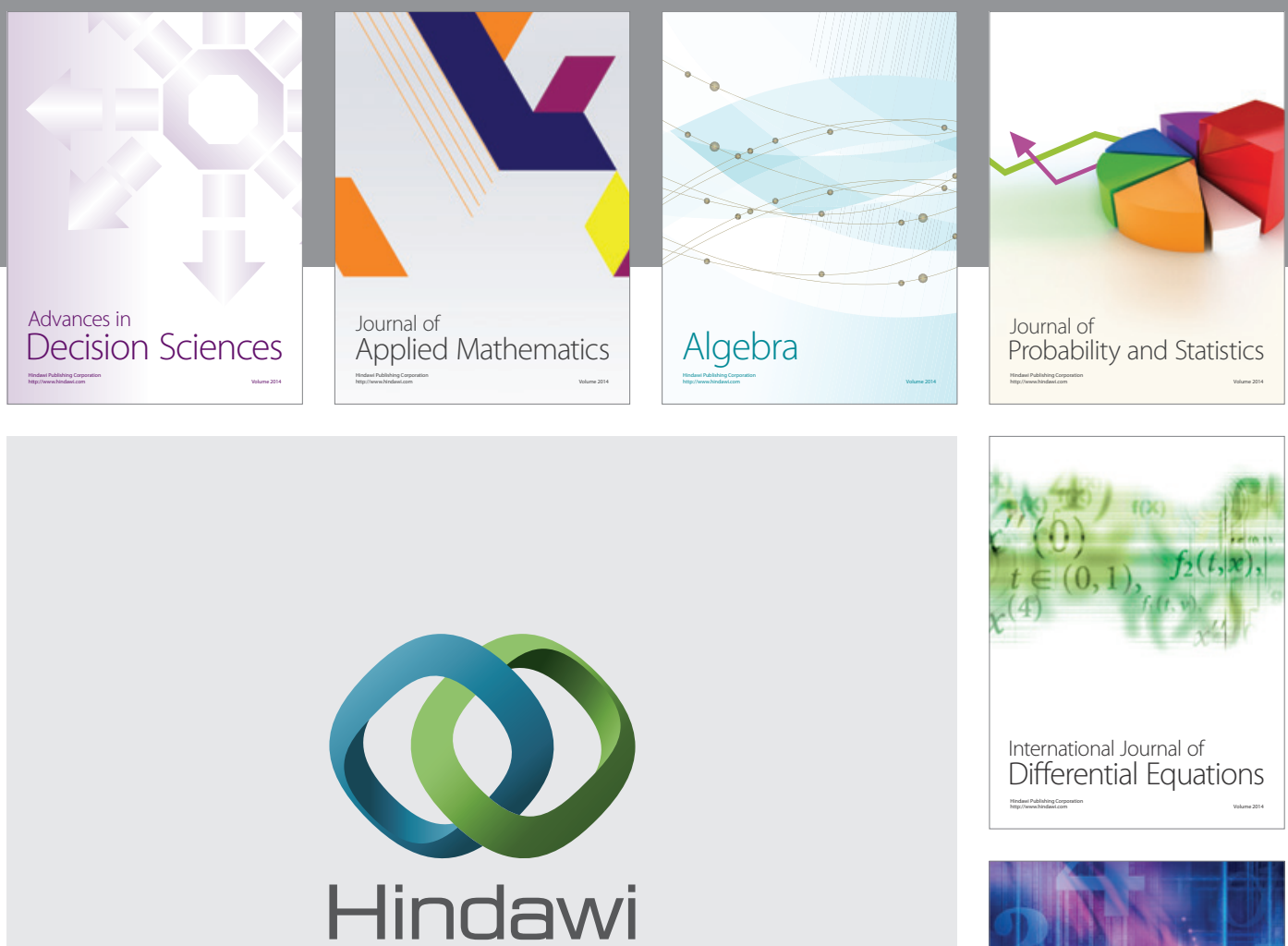

Submit your manuscripts at http://www.hindawi.com
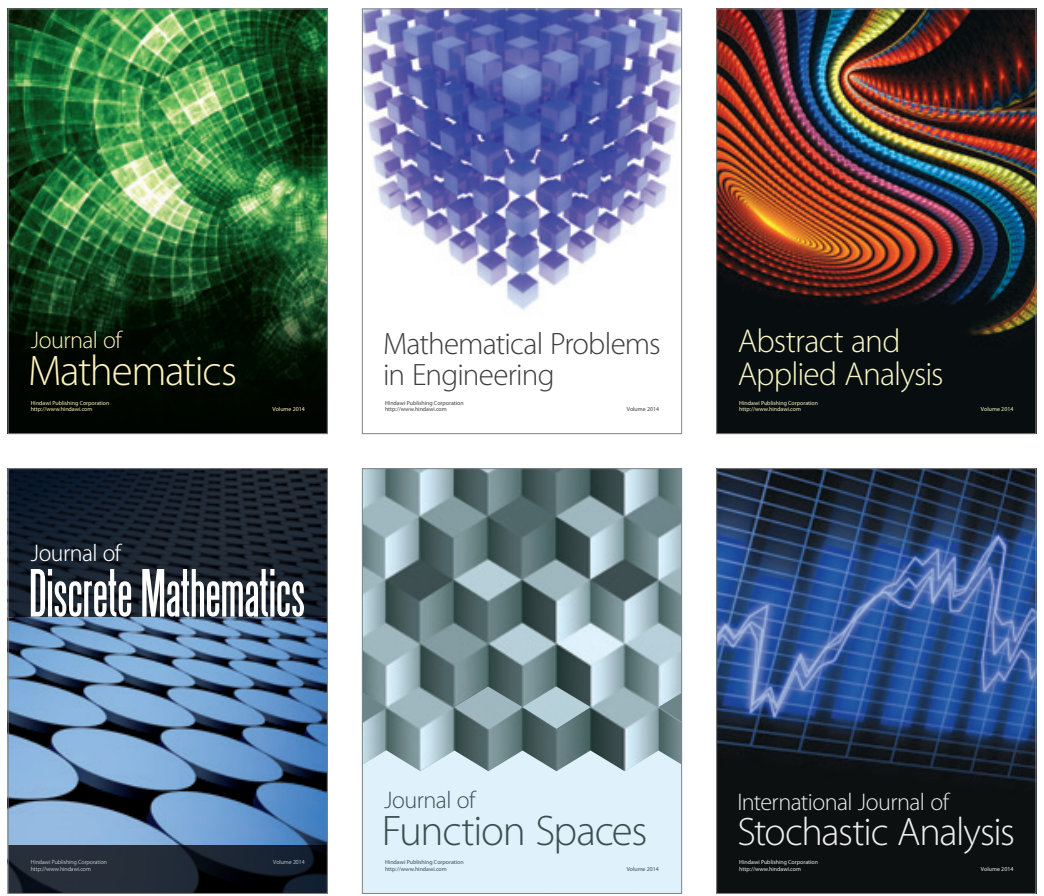

Journal of

Function Spaces

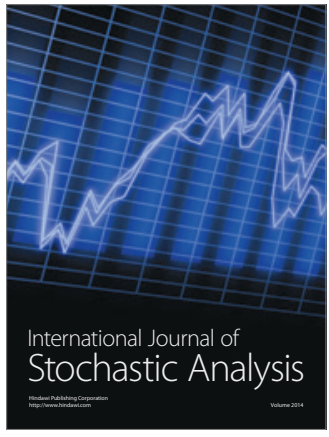

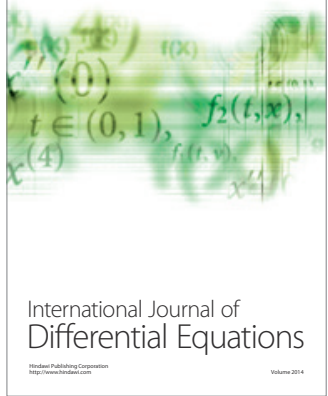
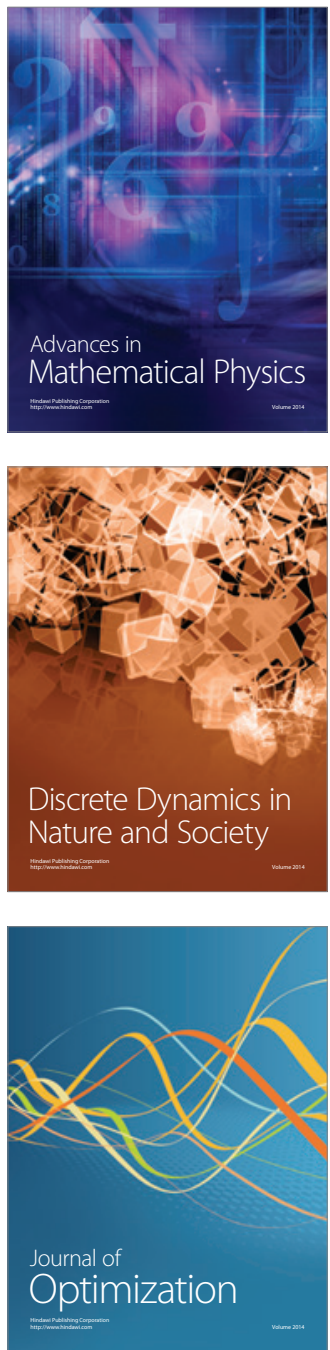